\title{
Mouse Models of Colorectal Cancer-Derived Circulating Tumor Cells
}

\author{
Lahiri Kanth Nanduri, Sebastían A, Jürgen Weitz and Sebastian Schölch*
}

Department of Gastrointestinal, Thoracic and Vascular Surgery, Medizinische Fakultät Carl Gustav Carus, Technische Universität Dresden, Dresden, Germany

\begin{abstract}
Metastasis is the major cause of death in colorectal cancer (CRC) patients. Circulating tumor cells (CTCs) are the biologic correlate of metastasis. Despite the clinical relevance of CTCs, there has been little progress in understanding their biology in detail; particularly the phenotype of CTCs with metastasis-forming capacities is still unknown. In this article we discuss the benefits and limitations of mouse models of CRC-derived CTCs.
\end{abstract}

Keywords: Colorectal cancer; Tumor cells; Metastasis; Primary tumor

\section{Colorectal Cancer}

Colorectal cancer (CRC) is one of the most common tumor diseases [1] and has high mortality and morbidity rates especially in metastatic stages [2]. The vast majority of CRC-related deaths are due to distant metastases, most prominently to the liver, rather than the primary tumor [2]. Metastasis is a multi-step process during which the cells develop a migratory phenotype and detach from the primary tumor, invade the local vasculature and survive in circulation, extravasate into distant organs and finally outgrow to manifest metastatic lesions $[3,4]$. The presence of circulating tumor cells (CTCs) in the blood stream of the patients indicates metastatic activity and is a negative prognostic factor in all stages of CRC [5-10].

Metastatic dissemination is a dynamic process requiring dramatic phenotypic alterations in order for the tumor cells to survive the challenges posed in circulation and by the microenvironment at the distant metastatic sites. Hence, CTCs require a considerable degree of plasticity in order to successfully complete the process of metastasis. As distant metastases are generally phenotypically similar to the primary tumor [11], most of these phenotypic changes must be reversible. Prominent examples of this plasticity are epithelial-to-mesenchymal transition (EMT), which CTCs undergo to gain a migratory phenotype by down-regulation of epithelial markers (E-cadherin, EpCAM) and upregulation of mesenchymal markers (N-cadherin, vimentin); and mesenchymal-to-epithelial transition (MET) in the target organ, ultimately resulting in epithelial metastases [12-17].

Although tumors shed millions of CTCs into circulation [18], in most patients only a few metastases arise during the course of the disease. Therefore, it is conceivable that only a very small fraction of CTCs is actually capable of forming distant metastases. It is these few CTCs with metastasis-forming capacities which must be identified and characterized in order to conceive novel anti-metastatic therapeutics. The phenotype of metastasis-forming CTCs has already been narrowed down in breast cancer-derived CTCs [19]; however, tens of thousands of cells had to be screened and thousands of metastasis-forming CTCs had to be injected into mice in order to induce metastases in these animals. As CTCs are a much rarer event in CRC than in breast cancer, such an approach seems hardly possible in CRC. Additionally, the still rather large amount of CTCs needed to induce tumors in mice indicates the need of further characterization in order to detect the real metastasis-forming CTC fraction. Therefore, despite a growing body of evidence demonstrating the clinical relevance of colorectal cancerderived CTCs, there is still urgent need for further molecular and functional characterization of CRC-derived CTCs. As the phenotype of CTCs always needs to be evaluated in knowledge of the primary tumor's phenotype, another obstacle of CTC research is the rare availability of both CTCs and tumor tissue simultaneously, which is only possible when CTCs are isolated intraoperatively [4].

One possible way of overcoming all above obstacles are mouse models. While it is difficult and in many cases impossible to obtain CTCs from patients along with the corresponding tumor tissue for comparative analysis, this is easily possible in mouse models. Also, mouse experiments can be easily repeated and reproduced, thus increasing the number of CTCs available for analysis. Furthermore, mice present similar physiological challenges for CTCs as humans as the tumor cells have to go through the same steps of the metastatic cascade as in humans, making mouse model-derived CTCs a promising model for human CTCs.

As a result of these considerations, we have recently established an orthotopic and highly metastatic mouse model of CRC [20]. We injected human CRC cell lines orthotopically into the caecum of immunodeficient NOD scid gamma (NSG) [21] mice to generate local tumors which produced distant metastases and CTCs with high reproducibility. We demonstrated the human HCT116 CRC line, among others tested, to reliably form distant metastases and CTCs in all tumor-bearing mice within 35 days of tumor cell inoculation. A workflow was established and validated to systematically analyze both the functional and molecular properties of CTCs, as shown in Figure 1. The resulting CTCs were cultured in vitro and proved tumorigenic when reinjected into mice.

qPCR expression profiling of the CTCs revealed a significant down regulation of epithelial markers (EpCAM, CK18/19, EGFR), which is well in line with previously published data about CTCs [4] and supports the theory of EMT during metastasis. In addition, adhesion molecules (CD166 and claudin-7) and along with Ki67, a cell proliferation marker, were downregulated in CTCs compared to bulk tumor cells, suggesting a metastatic phenotype along with a certain degree of dormancy of CTCs in circulation. The upregulation of markers generally considered cancer stem cell markers (BMI and DLG-7) on CTCs may indicate cancer stem-like cell driven CRC progression and metastasis. The same model, along with other models [22,23], has also been used to evaluate anti-metastatic drugs, successfully using the number of CTCs and

*Corresponding author: Sebastian Schölch, Department of Gastrointestinal, Thoracic and Vascular Surgery, Medizinische Fakultät Carl Gustav Carus, Technische Universität Dresden, Fetscherstr. 74, 01307 Dresden, Germany, Tel: +49(0)351/458-18941; E-mail: sebastian.schoelch@uniklinikum-dresden.de

Received July 11, 2016; Accepted July 14, 2016; Published July 19, 2016

Citation: Nanduri LK, García SA, Weitz J, Schölch S (2016) Mouse Models of Colorectal Cancer-Derived Circulating Tumor Cells. Med chem (Los Angeles) 6: 497-499. doi:10.4172/2161-0444.1000389

Copyright: (c) 2016 Nanduri LK, et al. This is an open-access article distributed under the terms of the Creative Commons Attribution License, which permits unrestricted use, distribution, and reproduction in any medium, provided the original author and source are credited. 


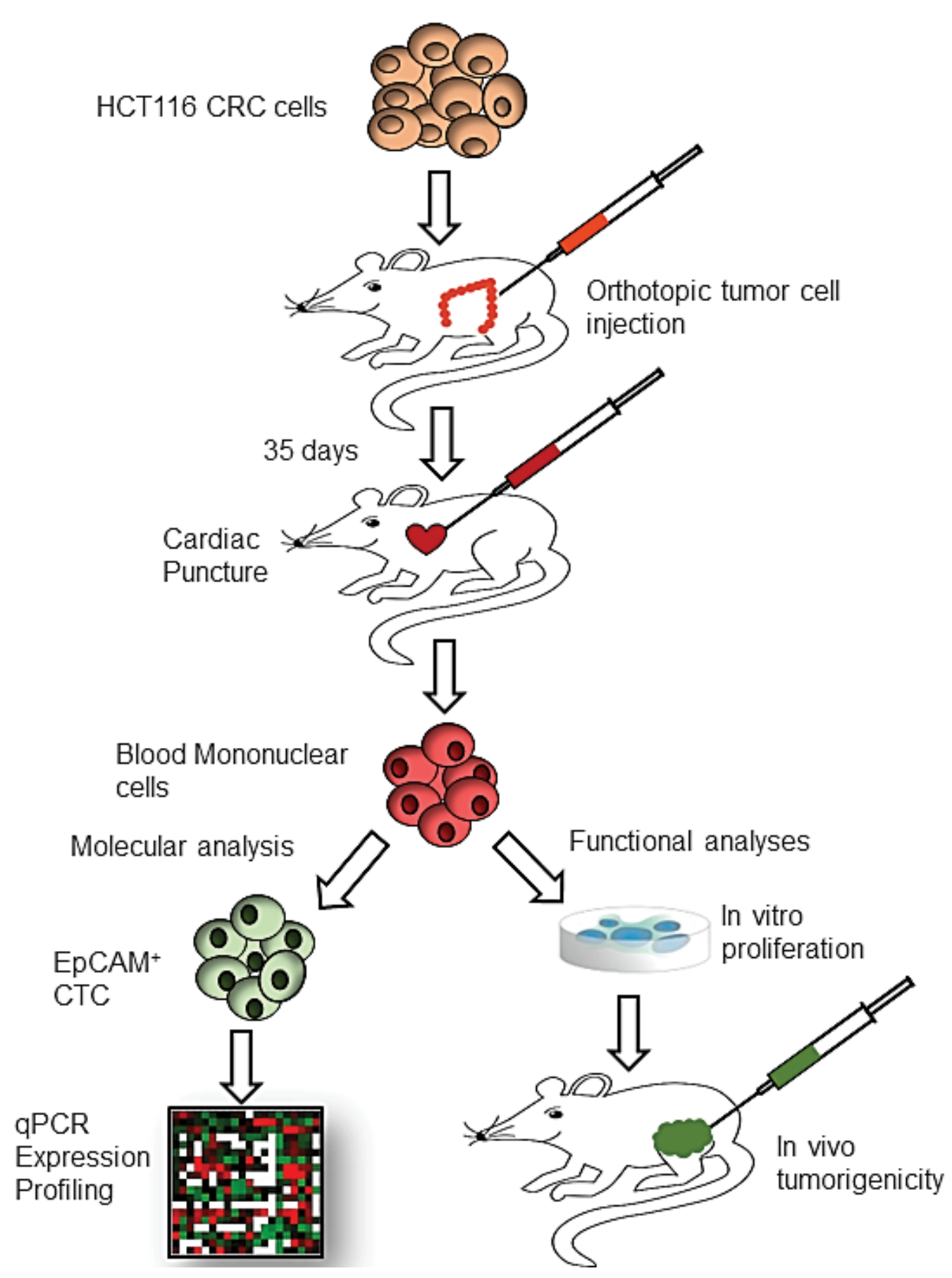

Figure 1: Schematic representation of the experimental workflow of CTC characterization. Adopted from Ref. [26].

distant metastases as end-points [24]. This further demonstrates the predictive value and practicability of the model.

Although orthotopic mouse models can serve as valuable models in understanding the biology of cancer progression, there are still some limitations. For example, recent studies on human CRC-derived CTCs demonstrated overexpression of CD47 along with downregulation of calreticulin on CTCs, a combination which is suggestive of an active immune escape program [4] of CTCs in circulation. In our mouse model, CTCs did not overexpress CD47 or downregulate calreticulin.
This may be explained by the model organism we used. NSG mice are highly immunodeficient; as there is no immunosurveillance in circulation, CTCs are not subjected to Mendelian selection for immune escape properties and therefore do not require such a phenotype. Another limitation of this and other models [25] is the the use of cell lines to induce tumors. Most cell lines have been cultured for decades, have thus adapted to the laboratory culture conditions and may not fully represent the disease. Moreover, the CRC cell lines currently available cannot represent the vast heterogeneity of CRC. Therefore, we require experimental models which mimic human CRC 
as close as possible both in terms of their mutational landscape and molecular progression. A possible solution to these limitations may be genetically engineered mouse models (GEMMs), in which tumors are induced within the mouse cells by genetic manipulation. The resulting tumors closely resemble their human counterparts and grow in immunocompetent hosts; therefore representing a more realistic mouse model [26]. However, these mouse models are much more cumbersome and cost-intensive than cell line-based mouse models. As a result, we expect both cell line-based and genetically engineered mouse models to be used parallelly in the future, depending on the aim of the given experiment.

In conclusion, mouse models of metastatic CRC are a valuable tool to study the biology of CRC-derived CTCs. The addition of genetically engineered mouse models may complement conventional cell linebased mouse models in the future, constituting a comprehensive set of tools in CTC research.

\section{References}

1. Siegel RL, Miller KD, Jemal A (2016) Cancer statistics, 2016. CA Cancer J Clin 66: 7-30.

2. Weitz J, Koch M, Debus J, Höhler T, Galle PR, et al. (2005) Colorectal cancer. Lancet 365: 153-165.

3. Nguyen DX, Bos PD, Massagué J (2009) Metastasis: from dissemination to organ-specific colonization. Nat Rev Cancer 9: 274-284.

4. Schölch S, García SA, Iwata N, Niemietz T, Betzler AM, et al. (2016) Circulating tumor cells exhibit stem cell characteristics in an orthotopic mouse model of colorectal cancer. Oncotarget, p: 8373.

5. Rahbari NN, Aigner M, Thorlund K, Mollberg N, Motschall E, et al. (2010) Meta-analysis Shows That Detection of Circulating Tumor Cells Indicates Poor Prognosis in Patients With Colorectal Cancer. Gastroenterology 138: 17141726.

6. Rahbari NN, Bork U, Kircher A, Nimitz T, Schölch S, et al. (2012) Compartmental differences of circulating tumor cells in colorectal cancer. Ann Surg Oncol 19 2195-2202.

7. Koch M, Kienle P, Kastrati D, Antolovic D, Schmidt J, et al. (2006) Prognostic impact of hematogenous tumor cell dissemination in patients with stage II colorectal cancer. Int J Cancer 118: 3072-3077.

8. Cohen SJ, Punt CJA, lannotti N, Saidman BH, Sabbath KD, et al. (2009) Prognostic significance of circulating tumor cells in patients with metastatic colorectal cancer. Ann Oncol 20: 1223-1229.

9. Bork U, Grützmann R, Rahbari NN, Schölch S, Distler M, et al. (2014) Prognostic relevance of minimal residual disease in colorectal cancer. World $\mathrm{J}$ Gastroenterol 20: 10296-10304.

10. Cohen SJ, Punt CJA, lannotti N, Saidman BH, Sabbath KD, et al. (2008) Relationship of Circulating Tumor Cells to Tumor Response, Progression-Free Survival, and Overall Survival in Patients With Metastatic Colorectal Cancer. JCO 26: 3213-3221.
11. Stange DE, Engel F, Longerich T, Koo BK, Koch M, et al. (2010) Expression of an ASCL2 related stem cell signature and IGF2 in colorectal cancer liver metastases with 11p15.5 gain. Gut 59: 1236-1244.

12. Palen K, Weber J, Dwinell MB, Johnson BD, Ramchandran R, et al. (2016) E-cadherin re-expression shows in vivo evidence for mesenchymal to epithelial transition in clonal metastatic breast tumor cells. Oncotarget, p: 9715.

13. Diepenbruck M, Christofori G (2016) Epithelial-mesenchymal transition (EMT) and metastasis: yes, no, maybe? Curr Opin Cell Biol 43: 7-13.

14. Vermaat JS, Nijman IJ, Koudijs MJ, Gerritse FL, Scherer SJ, et al. (2012) Primary Colorectal Cancers and Their Subsequent Hepatic Metastases Are Genetically Different: Implications for Selection of Patients for Targeted Treatment. Clin Cancer Res 18: 688-699.

15. Onstenk W, Sieuwerts AM, Mostert B, Lalmahomed Z, Bolt-de Vries JB, et al (2016) Molecular characteristics of circulating tumor cells resemble the liver metastasis more closely than the primary tumor in metastatic colorectal cancer. Oncotarget, p: 10175.

16. Rahbari NN, Bork U, Schölch S, Reissfelder C, Thorlund K, et al. (2016) Metastatic Spread Emerging From Liver Metastases of Colorectal Cancer: Does the Seed Leave the Soil Again? Ann Surg 263: 345-352.

17. Chang YS, di Tomaso E, McDonald DM, Jones R, Jain RK, et al. (2000) Mosaic blood vessels in tumors: frequency of cancer cells in contact with flowing blood. Proc Natl Acad Sci USA 97: 14608-14613.

18. Baccelli I, Schneeweiss A, Riethdorf S, Stenzinger A, Schillert A, et al. (2013) Identification of a population of blood circulating tumor cells from breast cance patients that initiates metastasis in a xenograft assay. Nat Biotechnol 31: 539544

19. Steinert G, Schölch S, Niemietz T, Iwata N, García SA, et al. (2014) Immune escape and survival mechanisms in circulating tumor cells of colorectal cancer. Cancer Res 74: 1694-1704.

20. Ito M, Hiramatsu H, Kobayashi K, Suzue K, Kawahata M, et al. (2002) $\mathrm{NOD/SCID/gamma(c)(null)} \mathrm{mouse:} \mathrm{an} \mathrm{excellent} \mathrm{recipient} \mathrm{mouse} \mathrm{model} \mathrm{for}$ engraftment of human cells. Blood 100: 3175-3182.

21. Schölch S, Rauber C, Tietz A, Rahbari NN, Bork U, et al. (2015) Radiotherapy combined with TLR7/8 activation induces strong immune responses against gastrointestinal tumors. Oncotarget 6: 4663-4676.

22. Schölch S, Rauber C, Weitz J, Koch M, Huber PE (2015) TLR activation and ionizing radiation induce strong immune responses against multiple tumor entities. Oncoimmunology 4: e1042201.

23. van Noort VV, Schölch S, Iskar M, Zeller G, Ostertag K, et al. (2014) Nove drug candidates for the treatment of metastatic colorectal cancer through global inverse gene expression profiling. Cancer Res 74: 5690-5699.

24. Tseng JY, Yang CY, Liang SC, Liu RS, Yang SH, et al. (2014) Interleukin-17A modulates circulating tumor cells in tumor draining vein of colorectal cancers and affects metastases. Clin Cancer Res 20: 2885-2897.

25. Sharpless NE, Depinho RA (2006) The mighty mouse: genetically engineered mouse models in cancer drug development. Nat Rev Drug Discov 5: 741-754.

26. Roper J, Hung KE (2012) Priceless GEMMs: genetically engineered mouse models for colorectal cancer drug development. Trends Pharmacol Sci 33: 449-455. 